Michelle Ziegler

\title{
Rettungsversuch im Jubiläumsjahr. Mauricio Kagels Aufarbeitung \\ der Beethoven-Rezeption in der Ludwig van-Werkgruppe (1970)
}

Jubiläumsjahre dienen vorzüglich dazu, die Einschätzung einer historischen Persönlichkeit zu überprüfen. Im Falle des schon zu seinen Lebzeiten verehrten Bonner Komponisten Ludwig van Beethoven besteht eine Fülle entsprechender Quellen, da sein Vermächtnis schon früh und nicht nur in den üblichen Fünfziger- und Zentenar-Intervallen öffentlich gewürdigt wurde - etwa mit der Einweihung des Bonner Denkmals zum 75. Geburtstag I845. Stand die Beethoven-Rezeption bis ins frühe 20. Jahrhundert im Licht überhöhender Affirmation und Vereinnahmung, wurden I920 zum I50. Geburtstag Beethovens auch kritische Stimmen wahrnehmbar. In der Zeit der Jugendbewegung zielte Ferruccio Busoni mit seinem Aufsatz »Was gab uns Beethoven ?« auf eine Neuausrichtung ab. Er forderte dazu auf, Beethovens Schaffen nicht mehr als das »zum Symbol gediehene Werk der musikalischen Menschlichkeit«zu missdeuten, sondern es von der "propagandistische[n] Geberde« zu befreien. ${ }^{\mathrm{I}}$ Doch die Erneuerungsversuche misslangen und schon das Jubiläumsjahr I927 brachte zumindest offiziell die »Restitution des sakralen Beethoven-Bildes «. ${ }^{2}$ Die politisch motivierte Traditionspflege führte bekanntlich im weiteren Verlauf des Jahrhunderts zu grauenhaften Missbräuchen. ${ }^{3}$ Dass diese gerade in Bezug auf Beethoven in den Nachkriegsjahrzehnten ihrer Aufarbeitung harrten, lieferte an sich schon Zündstoff für die Debatten im Jubiläumsjahr I970. Zudem wurden auch in der Musikszene im Anschluss an I968 antiautoritäre Positionen verhandelt, die ein klassisch-romantisches Schönheitsideal ablehnten, sich an bürgerlichen Traditionsträgern entzündeten und das Verhältnis zu den Komponisten der Vergangenheit nachhaltig veränderten. ${ }^{4}$ In diesem erhitzten Klima wurde Mauricio Kagels Beethoven-Film Ludwig van (I970) ${ }^{5}$ im Jubiläumsjahr als Provokation aufgefasst.

1 Ferruccio Busoni: Was gab uns Beethoven?, in: ders.: Von der Einheit der Musik. Verstreute Aufzeichnungen, Berlin 1922 (Max Hesses Handbücher, Bd. 76), S. 290-297, hier S. 293 und 295.

2 Helmut Loos: Das Beethoven-Jahr I970, in: ders.: E-Musik Kunstreligion der Moderne. Beethoven und andere Götter, Kassel 2017, S. 83-94, hier S. 93.

3 Vgl. unter anderem David B. Dennis: Beethoven in German Politics I8j0-I989, New Haven I996; Esteban Buch: La Neuvième de Beethoven, Paris I999, S. 23I-253.

4 Vgl. Beate Kutschke: The Celebration of Beethoven's Bicentennial in I970. The Antiauthoritarian Movement and Its Impact on Radical Avant-Garde and Postmodern Music in West Germany, in: The Musical Quarterly 93 (2010), S. 560-615.

5 Die zu Ludwig van erhaltenen Dokumente sind in mehreren Portionen in die Sammlung Mauricio Kagel der Paul Sacher Stiftung (PSS) in Basel aufgenommen worden. Für die freundliche Unterstützung und Beratung danke ich Matthias Kassel und Michèle Noirjean-Linder herzlich. 
Kagels Auseinandersetzung mit Beethoven beschränkte sich allerdings nicht aufden vom Westdeutschen Rundfunk (WDR) in Auftrag gegebenen Film Ludwig van. Ein Bericht. Als komplementäre mediale Umsetzungen dazu entstanden eine Partitur für ein Ensemblestück Ludwig van. Hommage von Beethoven, das vom Laboratorio Alea in Madrid in Auftrag gegeben und am 20. Januar I970 in Madrid uraufgeführt wurde, ${ }^{6}$ und eine SchallplattenAufnahme mit dem gleichen Titel.7 Insbesondere durch die Anweisungen in der Partitur regte Kagel zudem neue Auslegungen an, in denen Beethovens Werke als »Neue Musik« in einer "gesteigerten Subjektivität des Musikers « ${ }^{8}$ aufgeführt werden sollten. Er beschrieb seinen Beitrag zum Beethoven-Jahr selbst nicht als abgeschlossene Komposition, sondern als »Idee«: ^Anders als eine abgeschlossene Komposition oder ein work-in-progress ist >Ludwig van vor allem eine Idee. Sie versucht dem Interpreten zu sagen: die Musik der Vergangenheit soll auch als Musik der Gegenwart dargeboten werden.«9 Der Aufruhr, den Kagel insbesondere durch den Film verursachte, verstellte zunächst den Blick auf die vielschichtige Deutung, die Kagel in seiner ersten kompositorischen Auseinandersetzung mit einem Klassiker vornahm, und hat die Rezeption nachhaltig geprägt. Die wachsende historische Distanz erlaubt es indes, die Gewichtung zu verlagern, sodass heute Kagels Werkgruppe als kunstvolle wie auch assoziationsreiche Aufarbeitung der Beethoven-Rezeption und -Interpretation betrachtet werden kann. So werde ich zwar zunächst den Kontext der Jubiläumsfeierlichkeiten und die bereits vielfach beschriebene Nähe zu antiautoritären Positionen grob umreißen, ${ }^{\text {IO }}$ mich dann aber insbesondere Kagels Aktualisierung von Rezeptions- und Interpretationsmustern zuwenden. Dieser Fokus berücksichtigt Film, Partitur und Schallplatte als Werkgruppe gleichermaßen und stellt Ludwig van weniger als Werkgruppe über Beethoven selbst dar, sondern vielmehr als eine in mehreren Schritten perpetuierte Collage zur Rezeption und Interpretation von Beethovens Schaffen.

Das Beethoven-Jahr 1970 Der Großteil der Aktivitäten im Beethoven-Jahr I970 bewegte sich im vorhersehbaren Rahmen: Der Markt wurde mit einer »Fülle gewissenloser Beet-

6 Vgl. Kutschke: The Celebration of Beethoven's Bicentennial in I970, S.562 und Anm. 20.

7 Mauricio Kagel: Ludwig van. Ein Bericht [Film] (I969), in: The Mauricio Kagel Edition (DVD), München 2006; Mauricio Kagel: Ludwig van. Hommage von Beethoven [Partitur], Wien I970; Mauricio Kagel: Ludwig van [LP], Deutsche Grammophon I970, 2530 oI4.

8 Mauricio Kagel im Gespräch mit Karl Faust, Produzent der Deutschen Grammophon Gesellschaft, anlässlich der Schallplatten-Aufnahme im Dezember I969, veröffentlicht auf der Hülle der Schallplatte und im Vorwort zur Partitur, S. v-VI.

9 Ebd., S.vi.

10 Vgl. unter anderem Kutschke: The Celebration of Beethoven's Bicentennial in I970, und Sabine Sanio: Ig68 und die Avantgarde. Politisch-ästhetische Wechselwirkungen in der westlichen Welt, Sinzig 2008, insb. S. 85-88. 
hoven-Interpretationen " gesättigt, ${ }^{\text {II }}$ die Veranstalter programmierten Hommage-Konzerte und das Beethoven-Haus in Bonn lockte frisch restauriert zu einem Besuch der Geburtsstätte. Der Festakt in der Zürcher Tonhalle gibt einen Eindruck der Würdigungen, die auf Bewahrung bekannter Rezeptionsmuster ausgerichtet waren. So wurde für die Gedenkrede mit Emil Staiger ausgerechnet jener wertkonservative Literaturprofessor verpflichtet, der vier Jahre zuvor seinen Unwillen gegenüber der zeitgenössischen Literatur in einer polemischen Rede zum Ausdruck gebracht hatte, die seinen Ruf getrübt und den »Zürcher Literaturstreit« ausgelöst hatte. ${ }^{\text {I2 }}$ Staiger würdigte Beethoven als »düster über dem Chaos brütende[n] und sein Machtwort sprechende[n] Schöpfer.« «3 Obschon Staiger gleich zu Beginn festhielt, dass die noch vor einem halben Jahrhundert übliche Art, »an dem Menschen herumzurätseln«inzwischen »verdächtig«geworden sei, liest sich der Rest seiner Rede als Sammlung eben solcher Persönlichkeitsausdeutung und daran lose angeschlossener Rückschlüsse auf das Werk - wie in der Deutung von Beethovens »störrisch « zusammengepresstem Mund oder seiner »unerträglich bren-

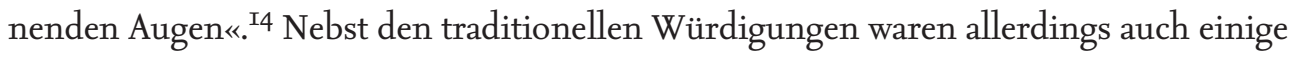
wenige kritische Stimmen zu vernehmen, die im Jahr der »Beethoven-Orgien etwas Nüchtern-Heilsames über seine Musik« zu vermitteln trachteten - so der Herausgeber des Bandes Beethoven 'jo mit Aufsätzen von Heinz-Klaus Metzger, Theodor W. Adorno, Hansjörg Pauli, Jacques Wildberger, Dieter Schnebel und Mauricio Kagel.15

Das Jubiläumsjahr I970 zeichnete sich zudem dadurch aus, dass mehrere etablierte Kulturinstitutionen Avantgarde-Komponisten in ihre Veranstaltungen involvierten. Nebst dem Auftrag des WDR an Kagel erhielt Karlheinz Stockhausen die Anfrage, einen Vortrag über Beethoven zu halten, worauf Stockhausen vorschlug, gemeinsam mit anderen Interpreten einen Abend lang »spielend über Beethovens Musik zu meditieren «. ${ }^{\mathrm{T}}{ }$

11 Hanspeter Krellmann: Song of Joy. Ein Rückblick auf das Beethovenjahr I970, in: Musica 25 (I97I), S. 9-II, hier S. 9.

12 Vgl. Emil Staiger: Literatur und Oeffentlichkeit. Eine Rede, in: Neue Zürcher Zeitung vom 2o. Dezember I966, Morgenausgabe, Blatt 5r/v; vgl. dazu unter anderem Joachim Rickes: Emil Staiger als Kritiker der Gegenwartsliteratur, in: Bewundert viel und viel gescholten. Der Germanist Emil Staiger (Igo8-I987), hg. von Joachim Rickes, Würzburg 2009, S. IOI-II4.

13 Emil Staiger: Beethoven. Eigenmacht und Gesetz, in: Neue Zürcher Zeitung vom 20. Dezember I970, S. 49 f., hier S. 50 .

14 Ebd., S. 49.

15 Mario Wildschütz: Nachbemerkung, in: Beethoven 'jo, Adorno, Kagel, Metzger, Pauli, Schnebel, Wildberger, hg. von Mario Wildschütz, Frankfurt a. M. I970, S. 62 f., hier S. 63.

16 Karlheinz Stockhausen: Kurzwellen mit Beethoven. Opus I970, in: ders.: Texte zur Musik I963-1970. Einführungen und Projekte, Kurse, Sendungen, Standpunkte, Nebennoten, hg. von Dieter Schnebel, Köln I97I (Texte zur Musik, Bd.3), S. I2If., hier S. I2I (Hervorhebung im Original). 
Anstelle der für Kurzwellen (I968) verwendeten Radiogeräte setzte er in Kurzwellen mit Beethoven (Opus I970) Tonbandgeräte mit elektronisch verfremdeten Beethoven-Aufnahmen ein. Der niederländische Komponist Louis Andriessen komponierte das Orchesterwerk Die neun Symphonien von Beethoven (De negen spmfonieën van Beethoven) (I970) für eine achtstündige Veranstaltung zum Abschluss des Holland Festivals I970. Andriessen zitierte aus allen neun Sinfonien in deren Reihenfolge, verfremdete sie und überlagerte sie ihrerseits mit Zitaten aus Jazz-, Rock- und Pop-Musik, der Internationalen und der holländischen Hymne. Wie Kagels Ludwig van basierten auch Stockhausens und Andriessens Jubiläumsbeiträge auf einer Collage von verfremdeter Musik Beethovens. Dabei setzte Stockhausen auf eine sanfte, »intuitive« Erneuerung der »gesamten Musik« Beethovens, ${ }^{17}$ während Andriessen mit seinem bewusst provozierenden Beitrag eine Erneuerung im Kulturbetrieb auslösen wollte. ${ }^{\text {I8 }}$

Kagels Film Ludwig van wurde im Rahmen der Wiener Festwochen uraufgeführt und vier Tage später im Fernsehen (WDR) ausgestrahlt. Die Stadt Wien profilierte sich neben Bonn als Hauptaustragungsort des Gedenkjahrs: Die Wiener Festspiele boten ein breites Angebot an Konzerten rund um Beethoven, während im Rathaus eine Beethoven-Ausstellung mit dem Titel »Die Flamme lodert« gezeigt wurde. Mitten in dieser Wiener Festspiel-Euphorie wurde Kagels Ludwig van als Affront aufgefasst, die Uraufführung von gehässigen Zwischenrufen begleitet. Hilde Spiel enervierte sich in der Frankfurter Allgemeinen Zeitung über die »Inhumanität des ganzen Unternehmens ${ }^{\text {}}{ }^{\text {I9 }}$ - mit Verweis auf die Sequenz im Zoo und die Großaufnahmen eines Elefantenhintern zur »Ode an die Freude«. Freilich bezog sich Kagel im Titel seines Filmes mit der Beschränkung auf den Vornamen betont jovial auf den großen Komponisten. ${ }^{20}$ Er ließ seine erleuchtende Flamme aus der Gosse emporlodern und baute weitere direkte Provokationen ein. Allerdings entgegnete er auf den Vorwurf, einen Anti-Beethoven-Film gedreht zu haben, dass ihm das Werk Beethovens so viel bedeute, dass er sich entschlossen habe, solche »Miss-

Ebd., S. I2I und I22.

18 Stephen Loy: Music, Activism and Tradition. Louis Andriessen's Nine Symphonies of Beethoven, in: Context 34 (2009), S. 15-34.

19 Hilde Spiel: Kagels Anti-Beethoven-Film in Wien uraufgeführt, zit. nach Melos 9 (r970), S.366f., hier S. 367 .

20

Der Titel Ludwig van erinnert auch an den Titelhelden aus Anthony Burgess' Roman A Clockwork Orange (1962), den Stanley Kubrick I97I verfilmte. Im Austausch mit Christian Brix verneinte Kagel allerdings 2004 einen entsprechenden Zusammenhang; vgl. Christian Brix: $»$ Ludwig van«. Zu Mauricio Kagels Beethoven-Film, Norderstedt 2004, S.35; Damien Ehrhardt: Ludwig van. De Kagel à Kubrick, in: Kubrick, les musiques, hg. von Brigitte Gauthier, Montpellier 2012 (Kubrick, les films, les musiques, Bd. 2), S. roI-III, hier S. Ior. 
verständnisse « in Kauf zu nehmen. ${ }^{2 \mathrm{I}}$ In einer frühen Notiz betitelte er sein Werk sogar als »Dankgesang an B.«. ${ }^{22}$

Zur Beethoven-Rezeption. Ein vielschichtiger »Dokumentationsbericht « Mit einer breiten Auseinandersetzung nahm Kagel in seiner »Kunst des Kommentars « ${ }^{23}$ nicht vornehmlich Beethoven, sondern die Beethoven-Rezeption im Vorfeld des Jubiläumsjahrs ins Visier. Bereits in einem frühen Exposé betitelte Kagel sein neues Werk mit »Dokumentationsbericht «. ${ }^{24}$ In einer groben Anlage definierte er unter den zwei Stichworten »Beethoven-Haus« und »Das Fernsehen« Möglichkeiten für vielschichtige Verweise und Assoziationen auf die Beethoven-Rezeption im Jahr 1970. ${ }^{25}$ Im ersten Teil des Filmes, der »Niederkunft Beethovens in seinem Jubeljahr « ${ }^{26}{ }^{26}$ konfrontiert Kagel Beethoven gleich selbst mit der Rezeption seines Werkes. Aus der Perspektive Beethovens - der Kameramann filmt mit einer Schulterkamera und ist gemäß Drehbuch mit Schnallenschuhen, Samthosen und Hörrohren ausgerüstet ${ }^{27}$ - werden die Ankunft in Bonn, ein Spaziergang durch die Innenstadt zum Geburtshaus Beethovens und eine Rheinfahrt gezeigt. Dabei wird Beethoven nicht nur auf Schritt und Tritt mit Interpretationen seiner Musik konfrontiert, sondern auch mit dem Kult um sein Schaffen und seine Person. In einem Schallplattenladen betrachtet er das Angebot von Einspielungen seiner Werke, in seinem Geburtshaus spiegeln die von Fluxus-Künstlern eingerichteten Räume die Absurditäten des Gedenkgeschäfts: In der Badewanne zerfallen Dieter Roths BeethovenBüsten aus Schmalz und Marzipan, in Ursula Burghardts metallverkleidetem Wohnzimmer sind alle Anzeichen von privatem Leben stilisiert und das mit Partiturausschnitten beklebte Musikzimmer, das Kagel nach dem Ausfall des tschechischen Künstlers Jiří Kolář selbst gestaltete, erscheint als virtueller Hallraum der Musik Beethovens. Im zweiten Teil des Filmes wird die narrative Linie gebrochen: Durch kurze Ansagen von Fern-

21 Mauricio Kagel/Felix Schmidt: Beethovens Erbe ist die moralische Aufrüstung, in: Der Spiegel vom 7. September 1970, S. 195-197, hier S. I95.

22 Mauricio Kagel: Notizen »Gedanken zur Komposition«(Ringbuch »Gedanken, Skizzen, Quellen«), Sammlung Mauricio Kagel, pss, [S.5].

23 Björn Heile: The Music of Mauricio Kagel, Aldershot 2006, S. 4.

24 Der Untertitel »Ein Dokumentationsbericht von Mauricio K.« steht auf einer Kopie des Exposés in der Sammlung Mauricio Kagel, ohne Untertitel als »Frühes Exposé« ist es abgedruckt in: Mauricio Kagel. Das filmische Werk I. I965-I985, hg. von Werner Klüppelholz und Lothar Prox, S. 89. Verzeichnis der Quellen zu Ludwig van in Knut Holtsträter: Mauricio Kagels musikalisches Werk. Der Komponist als Erzähler, Medienarrangeur und Sammler, Köln/Wien/Weimar 20ıо (Schriftenreihe der Hochschule für Musik Franz Liszt, Bd. 5), S. 297 f.

25 Mauricio Kagel: Skizze »Themen Ludwig van«, Sammlung Mauricio Kagel, pss.

26 Dieter Schnebel: Mauricio Kagel. Musik, Theater, Film, Köln I970, S. 258.

27 Mauricio Kagel: Drehbuch Ludwig van, Sammlung Mauricio Kagel, Pss, S. 4. 
sehmoderatorinnen entsteht eine Gliederung von Sequenzen, die sich an die nüchterne Präsentationsweise von Dokumentar- oder Schulfilmen halten. Hier folgen Verweise auf Beethovens Schwerhörigkeit, auf eine Meldung eines letzten vermeintlichen BeethovenNachfahren, auf die Technik des Klavierspiels, berühmte Interpretinnen und Interpreten und die damals bekannte Sendung»Frühschoppen«. Die beliebig wirkende Auswahl und dichte Montage dieser breiten Verweise potenzieren die Trivialität des Heroenkults.

Das Vorgehen deckt sich prinzipiell mit Kagels Kompositionsprozess für instrumentale Werke und Theater: Auf eine Phase des Sammelns und der Katalogisierung ${ }^{28}$ folgte ein Verarbeitungsprozess, in dem Kagel bewusst Offenheit und Vieldeutigkeit entstehen ließ. Notizen zu Büchern, die Kagel bei der Vorbereitung des Films konsultierte, belegen seine breit ausgerichtete Recherche insbesondere auch von nicht-authentischen Quellen. Indem Kagel diese Quellen im Film oft zerschnitt, neu montierte und dabei Bedeutungsebenen ergänzte oder wegließ, entstand ein unterschiedlich dichtes Assoziationsnetz. So konnte Knut Holtsträter aufzeigen, dass Kagel der Sequenz »Medizinisches Institut « ${ }^{29}$ ursprünglich eine Textstelle aus Theodor von Frimmels Buch Beethovens äußere Erscheinung (1905) ${ }^{30}$ unterlegen wollte, die dann aber wegfiel:

»Indem Kagel die Texte im Laufe der Produktion `wegmontiertes, wurden diese Bilder aber nun ihrer unmittelbaren Bedeutung beraubt. Sie verweisen nicht mehr auf den Text und auf das Thema des Films, nämlich Beethoven, sondern entfalten eine weitergehende Vieldeutigkeit bzw. Polysemie.. «1 ${ }^{\mathrm{I}}$

Die Offenheit führt ferner zu Verschiebungen der Gewichtung von Bild- und Klang-Ebene - ein mediales Wechselspiel, das Kagels Schaffen generell und seinen Beethoven-Film im Besonderen prägt.

Kagels Beethoven-Interpretationen Sowohl für den Film Ludwig van als auch für die Partitur und die Schallplatte verwendete Kagel ausschließlich Werke Beethovens. Die Funktion der Zitate unterscheidet sich jedoch. Für die Filmproduktion stand Kagel ein Kammerensemble mit Flöte, Oboe, Klarinette, Fagott, Horn, Trompete, Posaune, Gitarre, Streichern, Pauken und Klavier zur Verfügung. Dieses setzte er für klangliche Verfremdungen ein. Dazu Kagel:

Vgl. Matthias Kassel: Theatrum compositorum. Mauricio Kagels Materialsammlungen, in: Der Schall. Mauricio Kagels Instrumentarium, hg. von Michael Kunkel und Martina Papiro, Saarbrücken 2009, S. 23-29. Kagel: Das filmische Werk I. $\mathrm{Ig}_{95}-\mathrm{Ig} 85$, S. 90 .

30 Theodor von Frimmel: Beethovens äußere Erscheinung, München/Leipzig I905 (Beethoven Studien, Bd.I).

31 Knut Holtsträter: Kompositionsweisen in Mauricio Kagels filmischer Arbeit zu Ludwig van dargestellt an der Handschuhsequenz und dem Musikzimmer, in: »Alte« Musik und »neue« Medien, hg. von Jürgen Arndt und Werner Keil, Hildesheim 2003 (Diskordanzen, Bd. I4), S. 56-ז03, hier S. 62. 
"Der Idealfall wäre, Beethoven so aufzuführen, wie er hörte. Also: sschlecht‘. Das habe ich in der Filmfassung von >Ludwig van` kompositorisch versucht. Der Hauptgedanke war, seine Musik so umzuinstrumentieren, daß gewisse Klangbereiche und Frequenzen, die ein Tauber kaum oder verzerrt wahrnimmt, dementsprechend behandelt werden. « $3^{2}$

Neben den Neuinstrumentierungen legte Kagel verschiedene Aufnahmen des gleichen Stücks als »Demonstration der unterschiedlichen Interpretationen «33 übereinander und setzte moderne Spieltechniken ein, die Beethoven »sicher verwendet hätte, wenn er die weitere Entwicklung der Musiksprache erlebt hätte«.34 Dabei benutzte er für den Film weitgehend bekannte Werke Beethovens: Kaum eine populäre Klaviersonate fehlt(Mondschein- und Waldstein-Sonate, Pathétique, Der Sturm, Les adieux), daneben zitierte er Diabelli-Variationen, Neunte Sinfonie, Leonoren- und Coriolan-Ouvertüren. Die Bekanntheit der Stücke ist die Voraussetzung für Kagels Spiel mit Hörerwartungen, da die bearbeiteten Werke im Film stets bewusst in Beziehung zum Bild gebracht werden; sie ergänzen oder konterkarieren die Bild-Ebene.

In den unterschiedlichen Verbindungen zwischen Ton und Bild kommt ein kompositorisches Prinzip zur Anwendung, das Kagel unter den Stichworten »Synchronität« beziehungsweise »Asynchronität« bereits für den Film Duo (I968) umrissen hatte.35 Für Ludwig van erwog er für »Aktionen auf Instrumenten«, »Aktionen auf Gegenständen« und Bildindifferentes« unterschiedliche Modi. ${ }^{36}$ Die Verschiebungen sind beispielsweise in der Sequenz »Klavierpädagogik« zu erkennen, in der die Aktionen der Finger auf der Tastatur sich zunächst mit der erklingenden Sturm-Sonate op.3I Nr. 2 und der daran angehängten Coriolan-Ouvertüre in einer Bearbeitung für Klavier decken - wobei dem Interpreten an Armen und Beinen Schläuche und Kabel befestigt sind, die Werte für »Kraftleistung«, »Blutverlauf«, »koordinatorische Beschäftigungsneurose« messen. In dem Moment, da die Stimmen der Ouvertüre allmählich auseinandergeraten, geht auch der Zusammenhang zwischen Bild und Klang verloren, wozu die Kurve zur »Synchronität« sich glättet. Darüber hinaus überlagern sich auf Bild- und Klang-Ebene zudem oft unterschiedliche Bedeutungsschichten. In der Drehsequenz »Klavierabend Linda Claudius-Mann« etwa spielt der Pianist Klaus Lindemann als Elly Ney die Waldstein-Sonate op.53. Im Drehbuch sind einige dadaistische Effekte vorgesehen, wie die Bewegung des Busens der Pianistin, der sich selbständig »an den oberen Bildrand bege-

33 Mauricio Kagel: Notiz »Ludwig van, Rom« vom I2. April ı969, Kleines Ringbuch, Sammlung Mauricio Kagel, pss, [S.4].

34 Ebd.

35 Vgl. unter anderem Holtsträter: Kompositionsweisen in Mauricio Kagels filmischer Arbeit zu Ludwig van, S. 68.

36 Mauricio Kagel: Typoskript Bild/Ton, Sammlung Mauricio Kagel, pss. 

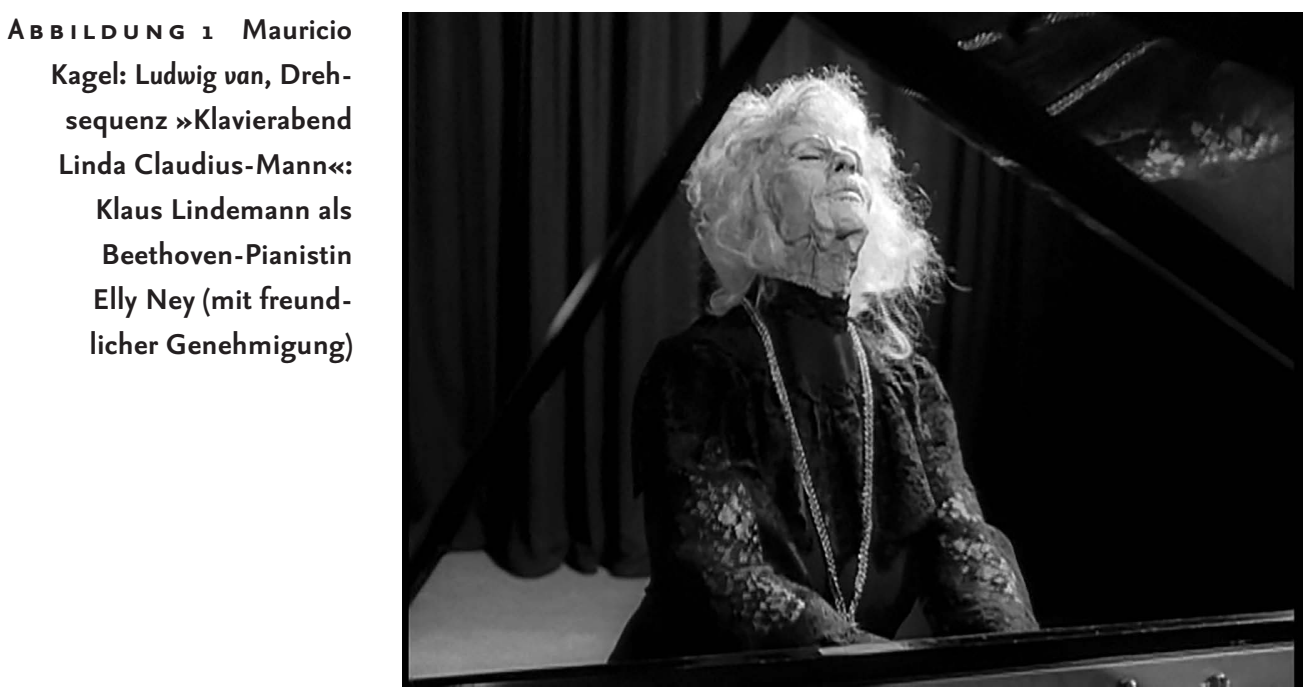

ben würde «, 37 und ein optisches Verwirrspiel mit an der Unterseite des Flügels montierten Fotografien der Klaviermechanik. Realisiert hat Kagel schließlich Verweise auf die Selbstinszenierung der deutschen Pianistin mit »ernster Beethoven-Pose « und »Beethoven-Frisur «, bei der die Haare bis unter die Klaviersaiten weiterwuchern. ${ }^{8}$ Der Bedeutungsgehalt der Bildebene wird durch die Verzerrung der gleichzeitig erklingenden Waldstein-Sonate assoziativ erweitert. Kagel fügte in die charakteristischen Achtel zu Beginn harte Akzente ein und verkehrte das Pianissimo in ein Fortissimo - was Elly Neys eigener Gewohnheit trotz ihrer Tendenz zur Überzeichnung widerspricht, allerdings in der militärischen Strenge lose an ihre Soldatenkonzerte im >Dritten Reich choralartige Seitenthema betonte er mit Bläsern. Mit der Verstärkung des Kontrasts zwischen den beiden Themen deutete Kagel die Sonate auf der Klangebene analytisch aus.

»Die Musiker mögen nun weitermachen« In den weiteren Umsetzungen seiner Ludwig van-»Idee« führte Kagel die Aktualisierung der Musik Beethovens durch Verfremdungen weiter. Die Bekanntheit der Stücke rückt dabei stärker in den Hintergrund. Die Partitur besteht aus einer Auswahl an Fotografien der Noten-Collage aus der »Musikzimmer«Sequenz des Filmes. Die Werke sind kaum erkennbar, da Kagel kurze Ausschnitte benutzte, die nur selten aus den Kopfsätzen von Sinfonien, Violin- und Klaviersonaten stammen. Die Erkennung im Ensemble-Stück wird dadurch erschwert, dass jede Kante

38 Beate Angelika Kraus: Elly Ney und Thérèse Wartel. Beethoven-Interpretation durch Pianistinnen eine Selbstverständlichkeit?, in: Der »männliche« und der »weibliche« Beethoven, hg. von Cornelia Bartsch, Beatrix Borchard und Rainer Cadenbach, Bonn 2003 (Schriften zur Beethoven-Forschung, Bd. 18), S. 429-447, hier S. 434. 

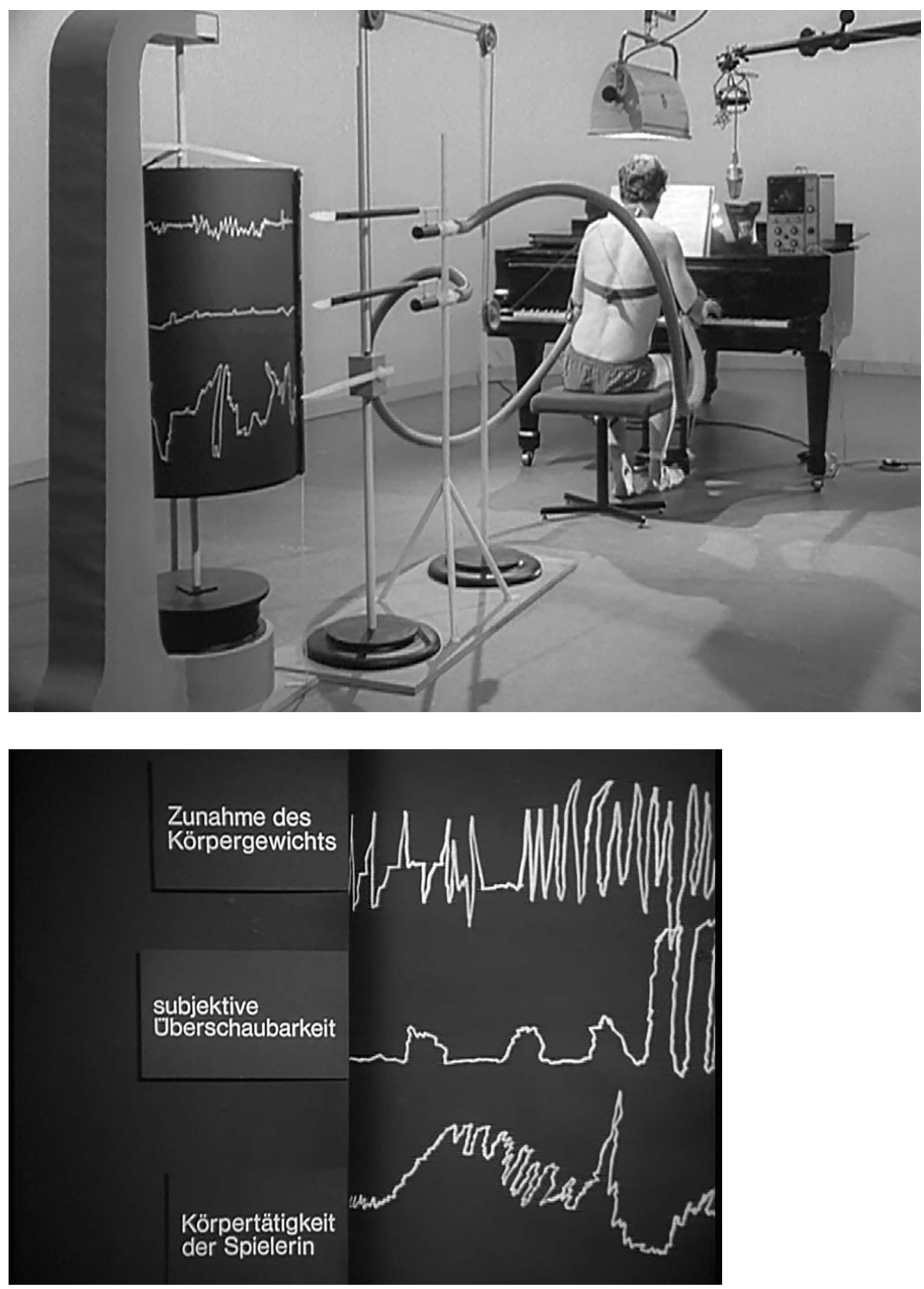

A в B ILDUNG 2 UND 3 Mauricio Kagel: Ludwig van, Drehsequenz »Klavierpädagogik«: Messung der Aktionen am Klavier (mit freundlicher Genehmigung) 
des Blattes als Unterkante dienen kann und die scharfen - oder die unscharfen-Bereiche der Vorlage verfremdet zu spielen sind. Dabei band Kagel die Interpreten stärker ein: Sie haben die zu spielenden Seiten auszuwählen, bestimmen den Ablauf, die Verfremdungen und das Tempo und können die Aufführung mit »Fragmenten anderer Werke Beethovens « ergänzen. ${ }^{39}$ Damit trat Kagel einen Teil der auktorialen Entscheidungen ab, was er am Schluss des Interviews im Vorwort der Partitur als Appell ausführte: »Dies alles gebe ich als Einleitung und Aufforderung: Die Musiker mögen nun weitermachen. «40

In seiner Umsetzung auf Schallplatte ging Kagel noch einen Schritt weiter. Gemäß einer Untersuchung Nikos Stavlas' hielt er sich hier nicht an die Ludwig van-Partitur, sondern erstellte unabhängig davon eine neue Collage mit Beethoven-Werken. ${ }^{4 \mathrm{I}}$ Er ließ ein Ensemble mit zwei Sängern, zwei Pianisten und einem Streichquartett längere und kürzere Ausschnitte aus den Streichquartetten, Violin-, Klavier- und Cellosonaten sowie aus Liedern spielen, die er sodann elektronisch bearbeitete und neu montierte. Das Konzept einer Verfremdung von Musik Beethovens deckt sich in den Grundzügen mit jenem für den Film und das Ensemble-Stück. Schon bei der Anfrage um Mitwirkung bei den Aufnahmen für die Schallplatte erklärte Kagel dem Pianisten Frederic Rzewski Ende November 1969: »Perfekteste Interpretationen sind nicht das, was ich anstrebe. Aber immerhin ... Es soll die Musik Beethoven [sic] und nicht die Noten hervortreten. Falsche Töne spielte B. auch. « ${ }^{42}$ Dabei griff Kagel nun vorsätzlich das Prinzip der Werktreue an, das zu jener Zeit als unangefochten galt:

"Unsere Auffassung von Werktreue - vor allem bei der Musik des I8. und I9. Jahrhunderts - ist erholungsbedürftig. Die krankhaften Anstrengungen, bekannte Musikstücke der Vergangenheit noch bekannter zu machen, hat in der Interpretation klassischer Musik eine Tendenz zur Mäßigung und Salonfähigkeit entwickelt. Statt einzelner Werke sollte heute die Essenz der Meister interpretiert werden. «43

In seiner Umsetzung für die Schallplatte näherte sich Kagel einer »Essenz« des Meisters, indem er anhand einer Montage kompositorisch stärker in die Musik eingriff, um deren Strukturen zu verdeutlichen. Er gliederte mit Kadenzen einen großen formalen Verlauf

39 Gemäss dem in der Sammlung Mauricio Kagel erhaltenen Aufführungsmaterial richtete Kagel auch selbst neue Fassungen ein (unter anderem für die London Sinfonietta und das Ensemble Intercontemporain). Dabei klebte er einerseits kurze Ausschnitte insbesondere aus Variationensätzen auf einzelne Seiten, die er kopierte, fügte aber auch ganze Partiturseiten etwa aus Klavierarrangements von Orchesterwerken (insbesondere von Franz Liszt) ein.

40 Kagel im Gespräch mit Faust, S. vi.

41 Nikos Stavlas: Reconstructing Beethoven. Mauricio Kagel's Ludwig van, London 2012, S. I24-I28, www. research.gold.ac.uk/7I5I/I/MUS_thesis_Stavlas_20I2.pdf(I2. Februar 20I8).

42 Mauricio Kagel an Frederic Rzewski, Brief vom 24. November I969, Sammlung Mauricio Kagel, pss.

43 Kagel im Gespräch mit Faust, S. v. 
von »Variationen«, montierte Stimmen aus unterschiedlichen Werken übereinander, fügte Pausen ein und betätigte Lautstärkeregler »zitternd und bebend«. ${ }^{44}$ Damit unternahm er im Studio eine Zergliederung und Neukomposition der Musik Beethovens, die er in Bezug auf das »Geistertrio «(Plattenseite A, Min Ir:35-I5:40) als »analytische Synthese dieser großartigen Musik« bezeichnete. ${ }^{45}$ Indem er sein Verfahren für die unterschiedlichen Medien anpasste und erweiterte, erreichte Kagel in der gesamten Ludwig vanGruppe unterschiedliche Grade der Aktualisierung.

Wurde Kagels Beethoven-Film im Jubiläumsjahr als Provokation aufgefasst, treten im Rückblick seine durchdachten »medialen Verschachtelungen « ${ }^{46}$ und die vielschichtige Auseinandersetzung mit Beethoven im gesamten Werkkomplex stärker hervor. Diese Verschiebung der Rezeption basiert auf einem längeren Prozess der Lockerung des Umgangs mit Autoritäten in der musikalischen Postmoderne. Kagels Ludwig van-Komplex stand im Jubiläumsjahr I970 an dessen Anfang. Im Schaffen des Komponisten selbst markiert es eine Wende hin zu einer vertieften Beschäftigung mit Werken der Vergangenheit in Kompositionen wie Variationen ohne Fuge(I97I/72) oder Sankt-Bach-Passion (1985), die parallel zu einer stärkeren Auseinandersetzung mit der musikalischen Tradition etwa in seinem Hauptwerk Staatstheater (I967/70) erfolgte. Der Ablösungsprozess prägte auch das darauffolgende Beethoven-Jahr I977, was etwa in Jürg Wyttenbachs musikszenischer Collage Beethoven: Sacré? - Sacré Beethoven! (I977) 47 - durchaus im Anschluss an Kagel - noch erkennbar wird. Bis heute ist an den Fundamenten im Umgang mit Klassikern bei gleichzeitigem Rückgang der Verbreitung klassischer Musik so stark gerüttelt worden, dass im nächsten Beethoven-Jahr 2020 wohl eher der Verlust eines entschwundenen Heroenbildes zutage treten wird.

44 Ebd., S. vi.

45 Ebd., S. v.

46 Matthias Kassel: Tonband - Bühne - Film. Mediale Stufen in Mauricio Kagels Antithese-Trilogie, in: RE-SET. Rückgriffe und Fortschreibungen in der Musik seit I90o, hg. von Simon Obert und Heidy Zimmermann, Mainz 2018, S. I57-166, hier S. I6ı.

47 Jürg Wyttenbach: Beethoven: Sacré? - Sacré Beethoven! Szenische Collage aus Texten, Musik, Aktionen, Film für Musiker, Sprecher u.a., in: Beethoven '77. Beiträge der Beethoven-Woche I977, hg. von Friedhelm Döhl, Zürich I979, S. I35-I64. 


\section{Inhalt}

Vorwort 8

INTERPRETATION - BEGRIFF, METHODE, PRAXIS

Laure Spaltenstein Interpretation als treue Übersetzung. Zur Frühgeschichte eines vieldeutigen Begriffs I5

Kai Köpp Von der Quelle zur Methode. Zum Entwurf einer historischen Interpretationsforschung $\quad 28$

Manuel Bärtsch >Interpretation‘. Beethovens Sonate A-Dur op. IOI in der Sicht von Eugen d'Albert und Frederic Lamond

Sebastian Bausch Klavierrollen als Interpretationsdokumente. Ein Erfahrungsbericht als Leitfaden für Einsteiger $\quad 7 \mathrm{I}$

Camilla Köhnken Beethoven-Auslegung zwischen Liszts »Deklamationsstil« und Bülows »Vivisektionsversuchen«. Auf den Spuren Liszt'scher Interpretationsideale in Hans von Bülows instruktiver Edition der Klaviersonaten Beethovens $\quad 92$

Neal Peres Da Costa Carl Reinecke's Performance of his Arrangement of the Second Movement from Mozart's Piano Concerto K. 488. Some Thoughts on Style and the Hidden Messages in Musical Notation

II4

Carolina Estrada Bascuñana Enrique Granados's Performance Style.

Visualising the Audible Evidence I5O

Lukas Näf Tempogestaltung in Weberns Sinfonie op. 2I I80

INTERPRETATION - AUFFÜHRUNGSGESCHICHTE

Christoph Moor "Ein so erklärtes Lieblingsstück der hiesigen Kunstfreunde«. Die Rezeptionsgeschichte der Jupiter-Sinfonie in Beethovens Wien

Luisa Klaus Objektive Bruckner-Interpretation? Zur Aufführung der Trio-Entwürfe für die Neunte Sinfonie I940 205

Chris Walton Von innen und von außen. Beethovens Neunte Sinfonie und die $>$ Wagner'sche< Dirigiertradition $\quad 2 \mathrm{I} 8$

Lena-Lisa Wüstendörfer Streit um Fidelio. Gustav Mahler und Felix Weingartner im Disput um Werktreue $\quad 238$

INTERMEZZO

Robert Levin Turning Point to Musical Modernity. Beethoven as Executor of the Legacy of C. P. E. Bach. Concert Lecture 249

INTERPRETATION - INSTRUMENTE, ANALYSE, EDITION

Martin Skamletz «Man hat diese Erweiterung des Tonumfanges seit ein paar Jahren an den Tasteninstrumenten sehr weit getrieben.« Der Umgang mit Grenzen beim späten Mozart und beim frühen Beethoven $\quad 263$ 
Stephan Zirwes Analyse und Interpretation. Adolph Bernhard Marx' Beethoven-Analysen 29I

Michael Ladenburger Was können wir aus Originalhandschriften von Beethoven für eine angemessene Interpretation lernen? 30I

Federica Rovelli Die Skizzenbuch-Ausgaben und ihre mögliche digitale Zukunft 3 I7

Johannes Gebauer Interpretationspraktische Stemmatik. Philologische Methoden in der Interpretationsforschung am Beispiel annotierter Notenausgaben von Rodes 24 Capricen und Beethovens Violinkonzert

John Rink Chopin Copying Chopin 349

Tomasz Herbut Alexander Goldenweiser und Beethovens Sonate op. IIo- eine Spurensuche 366

INTERPRETATION - KREATIVE ANEIGNUNG

Thomas Gartmann Beethoven als sein eigener Interpret. Gedanken zur Bearbeitung der Klaviersonate op. I4/ז für Streichquartett

Ivo Haag Die Sinfonien von Johannes Brahms - (auch) Klaviermusik?

Michael Lehner Das Orchester auf dem Klavier. Welte-Klavierrollen von Gustav Mahler und Richard Strauss als interpretationsanalytische Quellen

Roger Allen “That Is What Music Really Is". Richard Wagner's Reception of Beethoven's Piano Sonata in A Major Op. IOI $43 \mathrm{I}$

Daniel Allenbach Eine heroische< Neunte? Dmitri Schostakowitschs Neunte Sinfonie im Vergleich mit Ludwig van Beethoenvs Sinfonien Nr.3 und 9 44I

Simeon Thompson Beethoven und der Zweite Weltkrieg in der künstlerischen Reflexion der Nachkriegszeit. Stanley Kubricks A Clockwork Orange und Rolf Liebermanns Leonore 40/45 456

Michelle Ziegler Rettungsversuch im Jubiläumsjahr. Mauricio Kagels Aufarbeitung der Beethoven-Rezeption in der Ludwig van-Werkgruppe (I970) 465

Leo Dick Über den späten Beethoven zur >Postidentität،. Die Suche nach liminalen Räumen im gegenwärtigen Musiktheater am Beispiel von Matthias Rebstocks Berliner Produktion Büro für postidentisches Leben $\quad 476$

Elizabeth Waterhouse Choreographic Re-mix. William Forsythe's Trio (I996) and Beethoven's String Quartet No. I5 in a Minor Op. I32 487

László Stachó "Gradus ad Parnassum".

The Purgatory of Instrumental Technique

Namen-, Werk- und Ortsregister 522

Die Autorinnen und Autoren der Beiträge 


\section{Rund um BeEthoven \\ Interpretationsforschung heute • \\ Herausgegeben von Thomas}

Gartmann und Daniel Allenbach 


\section{MUSIKFORSCHUNG DER Hochschule der KÜnste Bern Herausgegeben von Martin Skamletz und Thomas Gartmann Band 14}


0 Dieses Buch ist in gedruckter Form im Dezember 2019 in erster Auflage in der Edition Argus in Schliengen/Markgräflerland erschienen. Gestaltet und gesetzt wurde es im Verlag aus der Seria und der SeriaSans, die von Martin Majoor im Jahre 2000 gezeichnet wurden. Gedruckt wurde es auf Eos, einem holzfreien, säurefreien, chlorfreien und alterungsbeständigen Werkdruckpapier der Papierfabrik Salzer im niederösterreichischen Sankt Pölten. Das Vorsatzpapier Caribic cherry wurde von Igepa in Hamburg geliefert. Rives Tradition, ein Recyclingpapier mit leichter Filznarbung, das für den Bezug des Umschlags verwendet wurde, stellt die Papierfabrik Arjo Wiggins in Issy-les-Moulineaux bei Paris her. Das Kapitalband mit rot-schwarzer Raupe lieferte die Firma Dr. Günther Kast aus Sonthofen im Oberallgäu, die auf technische Gewebe und Spezialfasererzeugnisse spezialisiert ist. Gedruckt und gebunden wurde das Buch von der Firma Bookstation im bayerischen Anzing. Im Internet finden Sie Informationen über das gesamte Verlagsprogramm unter www.editionargus.de, zum Institut Interpretation der Hochschule der Künste Bern unter www.hkb.bfh.ch/interpretation und www.hkb-interpretation.ch. Die Deutsche Nationalbibliothek verzeichnet diese Publikation in der Deutschen Nationalbibliografie; detaillierte bibliografische Daten sind im Internet über www.dnb.de abrufbar. (c) der zeitgleich erschienenen digitalen Version: die Autorinnen und Autoren, 20I9. Dieses Werk ist lizenziert unter einer Creative Commons Namensnennung-Nicht kommerziell 4.0 International Lizenz (CC BY-NC 4.o). DoI: https://doi.org/I0.26045/kp64-6I78 ISBN 978-3-93I264-94-9 